PROCEEDINGS OF THE

AMERICAN MATHEMATICAL SOCIETY

Volume 137, Number 3, March 2009, Pages 1103-1113

S 0002-9939(08)09525-7

Article electronically published on August 28, 2008

\title{
MORASSES AND FINITE SUPPORT ITERATIONS
}

\author{
BERNHARD IRRGANG
}

(Communicated by Julia Knight)

\begin{abstract}
We introduce a method of constructing a forcing along a simplified $(\kappa, 1)$-morass such that the forcing satisfies the $\kappa$-chain condition. Alternatively, this may be seen as a method to thin out a larger forcing to get a chain condition. As an application, we construct a ccc forcing that adds an $\omega_{2}$-Suslin tree. Related methods are Shelah's historic forcing and Todorcevic's $\rho$-functions.
\end{abstract}

\section{INTRODUCTION}

There are a number of consistency questions from two-cardinal combinatorics that were answered by Shelah's method of historic forcing or with the help of Todorcevic's $\rho$-functions: Can there exist a superatomic Boolean algebra with width $\omega$ and height $\omega_{2}$ (Baumgartner and Shelah [1, Martinez 13])? Is it possible that there is a function $f: \omega_{2} \times \omega_{2} \rightarrow \omega$, such that $f$ is non-constant on any rectangle with infinite sides (Todorcevic [24, 22])? Can one prove in $Z F C$ that every initially $\omega_{1-}$ compact $T_{3}$-space with countable tightness is already compact (Rabus [17], Juhasz and Soukup [10)? Is there consistently a forcing that satisfies ccc and adds a Kurepa tree (Jensen [8, Velickovic [26])? There are many more examples, but we cannot give a comprehensive overview here. In all cases there is a natural forcing with finite conditions that would solve the problem if it preserved cardinals. Since the conditions are finite, the suitable property of the forcing to guarantee cardinal preservation is the countable chain condition (ccc). Therefore one thins out the natural forcing in such a way that the remaining forcing satisfies ccc.

In the following, we will present the simplest case of a morass approach to such questions, i.e. to construct a ccc-forcing of size $\omega_{2}$. The basic idea is simple: We try to generalize iterated forcing with finite support. Classical iterated forcing with finite support as introduced by Solovay and Tennenbaum [18] works with continuous, commutative systems of complete embeddings of Boolean algebras or partial orders which are indexed along a well-order. The following holds: If every forcing of the system satisfies ccc, then the direct limit does also. So if e.g. all forcings of the system are countable, then its direct limit satisfies ccc. It will, however, have size $\leq \omega_{1}$ since it is a direct limit, while we want to construct a forcing of size $\omega_{2}$. To overcome this limitation, we will not consider a linear system indexed along a well-order, but rather a two-dimensional system indexed along a

Received by the editors October 6, 2006, and, in revised form, April 22, 2007, and February 1, 2008.

2000 Mathematics Subject Classification. Primary 03E05, 03E35, 03E40.

(C)2008 American Mathematical Society Reverts to public domain 28 years from publication 
simplified $\left(\omega_{1}, 1\right)$-morass. Since we want to obtain complete embeddings, we have to thin out the natural forcings. The way to do this follows very naturally from our approach. As an example of how the thinning out is done, we will construct a ccc forcing that adds an $\omega_{2}$-Suslin tree. The basic forcing we thin out is Tennenbaum's forcing for adding a Suslin tree with finite conditions.

Morasses were introduced by R. Jensen in the early 1970's to solve the cardinal transfer problem of model theory in $L$ (see e.g. Devlin [2]). For the proof of the gap2 transfer theorem a gap-1 morass is used. For higher-gap transfer theorems Jensen has developed so-called higher-gap morasses [9. In his Ph.D. thesis, the author generalized these to gaps of arbitrary size (see [7, 6, 5]). The theory of morasses is far developed and well examined. In particular, it is known how to construct morasses in $L$ [2, 4, 7, 5] and how to force them [19, 20]. Moreover, D. Velleman has defined so-called simplified morasses, along which morass constructions can be carried out more easily [27, 30, 29. Their existence is equivalent to the existence of ordinary morasses [3, 15]. The fact that the theory of morasses is so far developed is an advantage of the morass approach compared to historic forcing or $\rho$-functions. It allows straightforward generalizations to higher cardinals, while the conditions of the forcings can be kept finite.

While the general method presented here works for higher cardinals, we can in general not expect that the consistency statements can naively be extended by raising the cardinal parameters. For example, we force an $\omega_{2}$-Suslin tree along a gap-1 morass. An innocent generalization of the argument that the resulting tree has neither a branch nor an antichain of size $\omega_{2}$ would yield a tree on $\omega_{3}$ that has neither a branch nor an antichain of size $\omega_{2}$, which is of course impossible. The reason why this generalization does not work is that the gap-2 case yields a threedimensional construction. Therefore, the finite conditions of our forcing have to fit together appropriately in three directions instead of two directions, and that is impossible. So if and how a statement generalizes to higher gaps depend heavily on the concrete conditions.

The exact relationship between our approach and the methods of historic forcing and $\rho$-functions is an open question. The crucial step in our proof that chain conditions are preserved is the definition of the support of a condition. It resembles the definition of the "history" $t^{*}(\alpha)$ of an ordinal $\alpha$ given by Baumgartner and Shelah 1. However, there are various ways to set things up, and the definition of an FS system given below is just one of them. As far as $\rho$-functions are concerned, it is possible to directly read off a $\rho$-function from a simplified gap-1 morass. This is a result of C. Morgan in [14. It is, however, unclear how this relates to an approach as below which generalizes finite support iterations.

If $\mathbb{P}$ is the limit of a finite support iteration indexed along $\alpha$, then we can understand a $\mathbb{P}$-generic extension as being obtained successively in $\alpha$-many steps. Moreover, there are names for the forcings used in every step. This raises the question of whether a similar analysis is possible for a forcing which is constructed with our method. It would justify to call them FS iterations along morasses instead of FS systems along a morass, which was the name the author used until the referee pointed out the shortcoming concerning successive extensions.

We should also mention that besides historic forcing and $\rho$-functions there is another, quite different, method to prove consistencies in two-cardinal combinatorics. This is the method of forcing with models as side conditions or with side conditions 
in morasses. Models as side conditions were introduced by S. Todorcevic [23, 25], which was further developed by P. Koszmider [11] to side conditions in morasses. Unlike the other methods, it produces proper forcings which are usually not ccc. This is sometimes necessary. For example, Koszmider proved that if $\mathrm{CH}$ holds, then there is no ccc forcing that adds a sequence of $\omega_{2}$ many functions $f: \omega_{1} \rightarrow \omega_{1}$ which is ordered by strict domination mod finite. However, he is able to produce a proper forcing which adds such a sequence 11. More on the method can be found in Morgan's paper [16]. In the context of our approach, this raises the question of whether it is possible to define something like a countable support iteration along a morass.

\section{FS ITERATIONS}

Let $\mathbb{P}$ and $\mathbb{Q}$ be partial orders. A map $\sigma: \mathbb{P} \rightarrow \mathbb{Q}$ is called a complete embedding if

(1) $\forall p, p^{\prime} \in \mathbb{P}\left(p^{\prime} \leq p \rightarrow \sigma\left(p^{\prime}\right) \leq \sigma(p)\right)$,

(2) $\forall p, p^{\prime} \in \mathbb{P}\left(p\right.$ and $p^{\prime}$ are incompatible $\leftrightarrow \sigma(p)$ and $\sigma\left(p^{\prime}\right)$ are incompatible),

(3) $\forall q \in \mathbb{Q} \exists p \in \mathbb{P} \forall p^{\prime} \in \mathbb{P}\left(p^{\prime} \leq p \rightarrow\left(\sigma\left(p^{\prime}\right)\right.\right.$ and $q$ are compatible in $\left.\left.\mathbb{Q}\right)\right)$.

In (3), we call $p$ a reduction of $q$ to $\mathbb{P}$ with respect to $\sigma$.

If only (1) and (2) hold, we say that $\sigma$ is an embedding. If $\mathbb{P} \subseteq \mathbb{Q}$ such that the identity is an embedding, then we write $\mathbb{P} \subseteq \perp \mathbb{Q}$.

We say that $\mathbb{P} \subseteq \mathbb{Q}$ is completely contained in $\mathbb{Q}$ if $i d \uparrow \mathbb{P}: \mathbb{P} \rightarrow \mathbb{Q}$ is a complete embedding.

Let $\alpha \in \operatorname{Lim}$. A finite support (FS) iteration is a sequence $\left\langle\mathbb{P}_{\xi} \mid \xi \leq \alpha\right\rangle$ of partial orders together with a commutative system $\left\langle\sigma_{\xi \eta} \mid \xi<\eta \leq \alpha\right\rangle$ of complete embeddings $\sigma_{\xi \eta}: \mathbb{P}_{\xi} \rightarrow \mathbb{P}_{\eta}$ such that $\bigcup\left\{\sigma_{\xi \eta}\left[\mathbb{P}_{\xi}\right] \mid \xi<\eta\right\}=\mathbb{P}_{\eta}$ for limit $\eta$.

This is the original definition by Solovay and Tennenbaum in [18, except that they use Boolean algebras instead of partial orders. Moreover, it is well known that if $\sigma: \mathbb{P}_{1} \rightarrow \mathbb{P}_{2}$ is a complete embedding, then there is a $\mathbb{P}_{1}$-name $\dot{\mathbb{Q}}$ such that $\mathbb{P}_{2}$ and $\mathbb{P}_{1} * \dot{\mathbb{Q}}$ are forcing equivalent. This leads to the more common definition of FS iterations, where conditions are sequences of names. For the exact relationship between the two approaches, see Kunen's textbook [12, chapter VIII, §5, and exercise $\mathrm{K}$.

An important property of FS iterations is that they preserve the $\kappa$-cc:

Theorem 2.1. Let $\left\langle\left\langle\mathbb{P}_{\xi} \mid \xi \leq \alpha\right\rangle,\left\langle\sigma_{\xi \eta} \mid \xi<\eta \leq \alpha\right\rangle\right\rangle$ be an FS iteration. Assume that all $\mathbb{P}_{\xi}$ with $\xi<\alpha$ satisfy the $\kappa$-cc. Then $\mathbb{P}_{\alpha}$ also satisfies the $\kappa$-cc.

Proof. See the original article by Solovay and Tennenbaum [18] or any standard textbook.

\section{MORASSES}

A simplified $(\kappa, 1)$-morass is a structure $\mathfrak{M}=\left\langle\left\langle\theta_{\alpha} \mid \alpha \leq \kappa\right\rangle,\left\langle\mathfrak{F}_{\alpha \beta} \mid \alpha<\beta \leq \kappa\right\rangle\right\rangle$ satisfying the following conditions:

(P0) (a) $\theta_{0}=1, \theta_{\kappa}=\kappa^{+}, \forall \alpha<\kappa \quad 0<\theta_{\alpha}<\kappa$.

(b) $\mathfrak{F}_{\alpha \beta}$ is a set of order-preserving functions $f: \theta_{\alpha} \rightarrow \theta_{\beta}$.

(P1) $\left|\mathfrak{F}_{\alpha \beta}\right|<\kappa$ for all $\alpha<\beta<\kappa$.

(P2) If $\alpha<\beta<\gamma$, then $\mathfrak{F}_{\alpha \gamma}=\left\{f \circ g \mid f \in \mathfrak{F}_{\beta \gamma}, g \in \mathfrak{F}_{\alpha \beta}\right\}$.

(P3) If $\alpha<\kappa$, then $\mathfrak{F}_{\alpha, \alpha+1}=\left\{i d \uparrow \theta_{\alpha}, f_{\alpha}\right\}$, where $f_{\alpha}$ is such that $f_{\alpha}\lceil\delta=i d \uparrow \delta$ and $f_{\alpha}(\delta) \geq \theta_{\alpha}$ for some $\delta<\theta_{\alpha}$. 
(P4) If $\alpha \leq \kappa$ is a limit ordinal, $\beta_{1}, \beta_{2}<\alpha$ and $f_{1} \in \mathfrak{F}_{\beta_{1} \alpha}, f_{2} \in \mathfrak{F}_{\beta_{2} \alpha}$, then there are a $\beta_{1}, \beta_{2}<\gamma<\alpha, g \in \mathfrak{F}_{\gamma \alpha}$ and $h_{1} \in \mathfrak{F}_{\beta_{1} \gamma}, h_{2} \in \mathfrak{F}_{\beta_{2} \gamma}$ such that $f_{1}=g \circ h_{1}$ and $f_{2}=g \circ h_{2}$.

(P5) For all $\alpha>0, \theta_{\alpha}=\bigcup\left\{f\left[\theta_{\beta}\right] \mid \beta<\alpha, f \in \mathfrak{F}_{\beta \alpha}\right\}$.

Lemma 3.1. Let $\alpha<\beta \leq \kappa, \tau_{1}, \tau_{2}<\theta_{\alpha}, f_{1}, f_{2} \in \mathfrak{F}_{\alpha \beta}$ and $f_{1}\left(\tau_{1}\right)=f_{2}\left(\tau_{2}\right)$. Then $\tau_{1}=\tau_{2}$ and $f_{1}\left\lceil\tau_{1}=f_{2}\left\lceil\tau_{2}\right.\right.$.

Proof by induction over $\beta$. The base case of the induction is $\beta=\alpha+1$. Then the claim follows immediately from (P3). So assume that $\beta=\gamma+1$. Let, by (P2), $f_{i}=g_{i} \circ f_{i}^{\prime}$, where $f_{i}^{\prime} \in \mathfrak{F}_{\alpha \gamma}, g_{i} \in \mathfrak{F}_{\gamma \beta}$. Let $\tau_{i}^{\prime}=f_{i}^{\prime}\left(\tau_{i}\right)$. It follows as in the base case that $\tau_{1}^{\prime}=\tau_{2}^{\prime}$ and $f_{1}^{\prime} \uparrow \tau_{1}=f_{2}^{\prime} \uparrow \tau_{2}$. So, by the induction hypothesis, $\tau_{1}=\tau_{2}$ and $f_{1}^{\prime} \uparrow \tau_{1}=f_{2}^{\prime}\left\lceil\tau_{2}\right.$. Hence $f_{1}\left\lceil\tau_{1}=f_{2} \uparrow \tau_{2}\right.$.

Finally, let $\beta \in \mathrm{Lim}$. Then there exists by (P4) $\alpha<\gamma<\beta$ and $g \in \mathfrak{F}_{\gamma \beta}$ such that $f_{i}=g \circ f_{i}^{\prime}, f_{i}^{\prime} \in \mathfrak{F}_{\alpha \gamma}$. So $f_{1}^{\prime}\left(\tau_{1}\right)=f_{2}^{\prime}\left(\tau_{2}\right)$. Hence $\tau_{1}=\tau_{2}$ and $f_{1}^{\prime}\left\lceil\tau_{1}=f_{2}^{\prime}\left\lceil\tau_{2}\right.\right.$ by the induction hypothesis. Therefore $f_{1}\left\lceil\tau_{1}=f_{2} \uparrow \tau_{2}\right.$.

A simplified morass defines a tree $\langle T, \prec\rangle$ :

Let $T=\left\{\langle\alpha, \gamma\rangle \mid \alpha \leq \kappa, \gamma<\theta_{\alpha}\right\}$.

For $t=\langle\alpha, \nu\rangle \in T$ set $\alpha(t)=\alpha$ and $\nu(t)=\nu$.

Let $\langle\alpha, \nu\rangle \prec\langle\beta, \tau\rangle$ iff $\alpha<\beta$ and $f(\nu)=\tau$ for some $f \in \mathfrak{F}_{\alpha \beta}$.

If $s:=\langle\alpha, \nu\rangle \prec\langle\beta, \tau\rangle=: t, f \in \mathfrak{F}_{\alpha \beta}$ and $f(\nu)=\tau$, then $f \uparrow(\nu(s)+1)$ does not depend on $f$ by Lemma 3.1. So we may define $\pi_{s t}:=f \uparrow(\nu(s)+1)$.

Lemma 3.2. The following hold:

(a) $\prec$ is a tree, $h t_{T}(t)=\alpha(t)$.

(b) If $t_{0} \prec t_{1} \prec t_{2}$, then $\pi_{t_{0} t_{1}}=\pi_{t_{1} t_{2}} \circ \pi_{t_{0} t_{1}}$.

(c) Let $s \prec t$ and $\pi=\pi_{\text {st }}$. If $\pi\left(\nu^{\prime}\right)=\tau^{\prime}, s^{\prime}=\left\langle\alpha(s), \nu^{\prime}\right\rangle$ and $t^{\prime}=\left\langle\alpha(t), \tau^{\prime}\right\rangle$, then $s^{\prime} \prec t^{\prime}$ and $\pi_{s^{\prime} t^{\prime}}=\pi \uparrow\left(\nu^{\prime}+1\right)$.

(d) Let $\gamma \leq \kappa, \gamma \in$ Lim. Let $t \in T_{\gamma}$. Then $\nu(t)+1=\bigcup\left\{r n g\left(\pi_{s t}\right) \mid s \prec t\right\}$.

Proof. (a) First, we prove that $\prec$ is transitive. Let $\langle\alpha, \nu\rangle \prec\langle\beta, \tau\rangle$ be witnessed by $f \in \mathfrak{F}_{\alpha \beta}$ and $\langle\beta, \tau\rangle \prec\langle\gamma, \eta\rangle$ by $g \in \mathfrak{F}_{\alpha \beta}$. Set $h=g \circ f \in \mathfrak{F}_{\alpha \gamma}$ by (P2). Then $h(\nu)=\eta$. So $\langle\alpha, \nu\rangle \prec\langle\gamma, \eta\rangle$.

Now, let $\langle\alpha, \nu\rangle,\langle\beta, \tau\rangle \prec\langle\gamma, \eta\rangle$ and $\langle\alpha, \nu\rangle \neq\langle\beta, \tau\rangle$. It follows from Lemma 3.1 that $\alpha \neq \beta$. Let w.l.o.g. $\alpha<\beta$. Let $\langle\alpha, \nu\rangle \prec\langle\gamma, \eta\rangle$ be witnessed by $f \in \mathfrak{F}_{\alpha \gamma}$. By (P2) choose $g \in \mathfrak{F}_{\beta \gamma}$ and $h \in \mathfrak{F}_{\alpha \beta}$ such that $f=g \circ h$. Then $\langle\alpha, \nu\rangle \prec\langle\beta, h(\nu)\rangle \prec\langle\gamma, \eta\rangle$. However, $h(\nu)=\tau$ by Lemma 3.1. Hence $\langle\alpha, \nu\rangle \prec\langle\beta, \tau\rangle$. This proves that $\prec$ is a tree.

Finally, by (P2), for all $t \in T$ there is $s \prec t$ such that $\alpha(s)=\beta$ if $\beta<\alpha(t)$. This shows the second claim.

(b) follows immediately from (a) and the definition.

(c) Let $s \prec t$ be witnessed by $f \in \mathfrak{F}_{\alpha \beta}$. Then $s^{\prime} \prec t^{\prime}$ is also witnessed by $f$ and $\pi_{s^{\prime} t^{\prime}}=\pi \uparrow\left(\nu^{\prime}+1\right)$ holds by definition.

(d) It suffices to prove $\subseteq$. Let $\nu=\nu(t)$ and $\tau<\nu$. By (P5) choose $\alpha_{1}, \alpha_{2}<\gamma$ and $f_{i} \in \mathfrak{F}_{\alpha_{i} \gamma}$ such that $\tau \in r n g\left(f_{1}\right)$ and $\nu \in r n g\left(f_{2}\right)$. By (P4) choose $\beta$ such that $\alpha_{1}, \alpha_{2}<\beta<\gamma$ and $f_{i}^{\prime} \in \mathfrak{F}_{\alpha_{i} \beta}, g \in \mathfrak{F}_{\beta \gamma}$, where $f_{i}=g \circ f_{i}^{\prime}$. Then $\tau, \nu \in \operatorname{rng}(g)$. So let $g(\bar{\tau})=\tau$ and $g(\bar{\nu})=\nu$. Hence $\bar{\tau}<\bar{\nu}$, since $g$ is order-preserving. Let $s=\langle\beta, \bar{\nu}\rangle$. Then $s \prec t$ and $\pi_{s t}(\bar{\tau})=\tau$.

Lemma 3.3. Let $\alpha<\beta \leq \kappa$. Then $i d \uparrow \theta_{\alpha} \in \mathfrak{F}_{\alpha \beta}$. 
Proof by induction on $\beta$. The base case of the induction is $\beta=\alpha+1$. Then the claim is part of (P3). So assume that $\beta=\gamma+1$. By the induction hypothesis, $i d \uparrow \theta_{\alpha} \in \mathfrak{F}_{\alpha \gamma}$. By $(\mathrm{P} 3), i d \uparrow \theta_{\gamma} \in \mathfrak{F}_{\gamma \beta}$. Hence $i d \uparrow \theta_{\alpha}=\left(i d \uparrow \theta_{\gamma}\right) \circ\left(i d \uparrow \theta_{\alpha}\right) \in \mathfrak{F}_{\alpha \beta}$ by $(\mathrm{P} 2)$.

Finally, let $\beta \in \operatorname{Lim}$. Assume towards a contradiction that $i d \uparrow \theta_{\alpha} \notin \mathfrak{F}_{\alpha \beta}$. Let $f \in \mathfrak{F}_{\alpha \beta}$ be such that $\sup \left(f\left[\theta_{\alpha}\right]\right)$ is minimal. Since $f \neq i d \uparrow \theta_{\alpha}$, there are $\nu<\tau \in \theta_{\beta}$ such that $\nu \notin r n g(f)$ and $\tau \in r n g(f)$. Let $t=\langle\beta, \tau\rangle$. By Lemma 3.2 (d), there is an $s \prec t$ such that $\nu \in r n g\left(\pi_{s t}\right)$. Let $s=\langle\gamma+1, \bar{\tau}\rangle$ be the minimal such $s$. Let $\nu=\pi_{s t}(\bar{\nu})$. Furthermore, let $f=f_{3} \circ f_{2} \circ f_{1}$, where $f_{3} \in \mathfrak{F}_{\gamma+1, \beta}$, $f_{2} \in \mathfrak{F}_{\gamma, \gamma+1}$ and $f_{1} \in \mathfrak{F}_{\alpha \gamma}$. Then $\pi_{s t}=f_{3}\lceil\bar{\tau}+1$. Hence by the minimality of $s$, $f_{2} \neq i d \uparrow \theta_{\gamma}, \bar{\nu}<\theta_{\gamma}$ and $\bar{\tau} \geq \theta_{\gamma}$. Define $g:=f_{3} \circ\left(i d \uparrow \theta_{\gamma}\right) \circ f_{1}$. Then $g \in \mathfrak{F}_{\alpha \gamma}$ by (P2) and $r n g(g) \subseteq f_{3}\left[\theta_{\gamma}\right] \subseteq f_{3}(\bar{\tau})=\tau$. Hence $\sup \left(f\left[\theta_{\alpha}\right]\right)$ was not minimal. Contradiction!

Theorem 3.4. (a) If $V=L$, then there is a simplified $(\kappa, 1)$-morass for all regular $\kappa>\omega$.

(b) If $\kappa$ is an uncountable regular cardinal such that $\kappa^{+}$is not inaccessible in $L$, then there is a simplified $(\kappa, 1)$-morass.

(c) For every regular $\kappa>\omega$, there is a $\kappa$-complete (i.e. every decreasing sequence of length $<\kappa$ has a lower bound) forcing $\mathbb{P}$ satisfying $\kappa^{+}$-cc such that $\mathbb{P} \Vdash$ (there is a simplified $(\kappa, 1)$-morass $)$.

Proof. (a) See Devlin [2, VIII 2 and 4, or Velleman 27.

(b) See Devlin [2], VIII 4 and exercise 6, or Velleman [28].

(c) See Velleman [27.

\section{FS SYSTEMS ALONG MORASSES}

Let $\mathfrak{M}$ be a simplified $(\kappa, 1)$-morass. We want to define a generalization of an FS iteration which is not indexed along an ordinal but along $\mathfrak{M}$. One way of doing this is the following definition:

We say that $\left\langle\left\langle\mathbb{P}_{\eta} \mid \eta \leq \kappa^{+}\right\rangle,\left\langle\sigma_{s t} \mid s \prec t\right\rangle,\left\langle e_{\alpha} \mid \alpha<\kappa\right\rangle\right\rangle$ is an FS system along $\mathfrak{M}$ if the following conditions hold:

(FS1) $\left\langle\mathbb{P}_{\eta} \mid \eta \leq \kappa^{+}\right\rangle$is a sequence of partial orders such that $\mathbb{P}_{\eta} \subseteq_{\perp} \mathbb{P}_{\nu}$ if $\eta \leq \nu$ and $\mathbb{P}_{\lambda}=\bigcup\left\{\mathbb{P}_{\eta} \mid \eta<\lambda\right\}$ for $\lambda \in$ Lim.

(FS2) $\left\langle\sigma_{s t} \mid s \prec t\right\rangle$ is a commutative system of injective embeddings $\sigma_{s t}$ : $\mathbb{P}_{\nu(s)+1} \rightarrow \mathbb{P}_{\nu(t)+1}$ such that if $t$ is a limit point in $\prec$, then

$$
\mathbb{P}_{\nu(t)+1}=\bigcup\left\{\sigma_{s t}\left[\mathbb{P}_{\nu(s)+1}\right] \mid s \prec t\right\} .
$$

(FS3) $e_{\alpha}: \mathbb{P}_{\theta_{\alpha+1}} \rightarrow \mathbb{P}_{\theta_{\alpha}}$.

(FS4) Let $s \prec t$ and $\pi=\pi_{s t}$. If $\pi\left(\nu^{\prime}\right)=\tau^{\prime}, s^{\prime}=\left\langle\alpha(s), \nu^{\prime}\right\rangle$ and $t^{\prime}=\left\langle\alpha(t), \tau^{\prime}\right\rangle$, then $\sigma_{s t}: \mathbb{P}_{\nu(s)+1} \rightarrow \mathbb{P}_{\nu(t)+1}$ extends $\sigma_{s^{\prime} t^{\prime}}: \mathbb{P}_{\nu^{\prime}+1} \rightarrow \mathbb{P}_{\tau^{\prime}+1}$.

Hence for $f \in \mathfrak{F}_{\alpha \beta}$, we may define $\sigma_{f}=\bigcup\left\{\sigma_{s t} \mid s=\langle\alpha, \nu\rangle, t=\langle\beta, f(\nu)\rangle\right\}$.

(FS5) If $\pi_{s t}=i d \uparrow \nu(s)+1$, then $\sigma_{s t}=i d \uparrow \mathbb{P}_{\nu(s)+1}$.

(FS6)(a) If $\alpha<\kappa$, then $\mathbb{P}_{\theta_{\alpha}}$ is completely contained in $\mathbb{P}_{\theta_{\alpha+1}}$ in such a way that $e_{\alpha}(p)$ is a reduction of $p \in \mathbb{P}_{\theta_{\alpha+1}}$.

(b) If $\alpha<\kappa$, then $\sigma_{\alpha}:=\sigma_{f_{\alpha}}: \mathbb{P}_{\theta_{\alpha}} \rightarrow \mathbb{P}_{\theta_{\alpha+1}}$ is a complete embedding such that $e_{\alpha}(p)$ is a reduction of $p \in \mathbb{P}_{\theta_{\alpha+1}}$.

(FS7)(a) If $\alpha<\kappa$ and $p \in \mathbb{P}_{\theta_{\alpha}}$, then $e_{\alpha}(p)=p$.

(b) If $\alpha<\kappa$ and $p \in \operatorname{rng}\left(\sigma_{\alpha}\right)$, then $e_{\alpha}(p)=\sigma_{\alpha}^{-1}(p)$. 
To simplify notation, set $\mathbb{P}:=\mathbb{P}_{\kappa^{+}}$.

Unlike the case of FS iterations, it is unclear how a generic extension with respect to $\mathbb{P}_{\kappa^{+}}$can be viewed as being obtained by successive extensions. This would justify calling an FS system along $\mathfrak{M}$ an FS iteration along $\mathfrak{M}$.

However, as in the case of FS iterations it is sometimes more convenient to represent $\mathbb{P}$ as a set of functions $p^{*}: \kappa \rightarrow V$ such that $p^{*}(\alpha) \in \mathbb{P}_{\theta_{\alpha}}$ for all $\alpha<\kappa$.

To define such a function $p^{*}$ from $p \in \mathbb{P}$ set recursively

$$
\begin{aligned}
& p_{0}=p, \\
& \nu_{n}(p)=\min \left\{\eta \mid p_{n} \in \mathbb{P}_{\eta+1}\right\}, \\
& t_{n}(p)=\left\langle\kappa, \nu_{n}(p)\right\rangle, \\
& p^{(n)}(\alpha)=\sigma_{s t}^{-1}\left(p_{n}\right) \text { if } s \in T_{\alpha}, s \prec t_{n}(p) \text { and } p_{n} \in \operatorname{rng}\left(\sigma_{s t}\right) .
\end{aligned}
$$

Note that, by Lemma 3.2 (a), $s$ is uniquely determined by $\alpha$ and $t_{n}(p)$. Hence we really define a function. Set

$\gamma_{n}(p)=\min \left(\operatorname{dom}\left(p^{(n)}\right)\right)$.

By (FS2), $\gamma_{n}(p)$ is a successor ordinal or 0 . Hence, if $\gamma_{n}(p) \neq 0$, we may define

$p_{n+1}=e_{\gamma_{n}(p)-1}\left(p^{(n)}\left(\gamma_{n}(p)\right)\right)$.

If $\gamma_{n}(p)=0$, we let $p_{n+1}$ be undefined.

Finally, set $p^{*}=\bigcup\left\{p^{(n)} \uparrow\left[\gamma_{n}(p), \gamma_{n-1}(p)[\mid n \in \omega\}\right.\right.$ where $\gamma_{-1}(p)=\kappa$.

Note: If $n>0$ and $\alpha \in\left[\gamma_{n}(p), \gamma_{n-1}(p)\left[\right.\right.$, then $p^{*}(\alpha)=\sigma_{s \bar{t}}^{-1}\left(p_{n}\right)$ where $\bar{t}=$ $\left\langle\gamma_{n}(p)-1, \nu_{n}(p)\right\rangle$, because $p^{*}(\alpha)=p^{(n)}(\alpha)=\sigma_{s t}^{-1}\left(p_{n}\right)=\left(\sigma_{\bar{t} t} \circ \sigma_{s \bar{t}}\right)^{-1}\left(p_{n}\right)=\sigma_{s \bar{t}}\left(p_{n}\right)$, where the first two equalities are just the definitions of $p^{*}$ and $p^{(n)}$. For the third equality note that $\bar{t} \prec t$ since $i d \uparrow \theta_{\alpha} \in \mathfrak{F}_{\alpha \beta}$ for all $\alpha<\beta \leq \kappa$ by Lemma 3.3. So the equality follows from the commutativity of $\left\langle\sigma_{s t} \mid s \prec t\right\rangle$. The last equality holds by (FS5).

It follows from the previous observation that $\left\langle\gamma_{n}(p) \mid n \in \omega\right\rangle$ is decreasing. So the recursive definition above breaks down at some point, i.e. $\gamma_{n}(p)=0$ for some $n \in \omega$. However, that is good news because of the following.

The support of $p$ is defined by $\operatorname{supp}(p)=\left\{\gamma_{n}(p) \mid n \in \omega\right\}$. Hence $\operatorname{supp}(p)$ is finite.

Lemma 4.1. If $p^{*}(\alpha)$ and $q^{*}(\alpha)$ are compatible for $\alpha=\max (\operatorname{supp}(p) \cap \operatorname{supp}(q))$, then $p$ and $q$ are compatible.

Proof. Suppose that $p$ and $q$ are incompatible. Without loss of generality let $\nu:=$ $\min \left\{\eta \mid p \in \mathbb{P}_{\eta+1}\right\} \leq \min \left\{\eta \mid q \in \mathbb{P}_{\eta+1}\right\}=: \tau$. Set $s=\langle\kappa, \nu\rangle$ and $t=\langle\kappa, \tau\rangle$. Let $t^{\prime} \prec t$ be minimal such that $\nu \in r n g\left(\pi_{t^{\prime} t}\right)$ and $p, q \in r n g\left(\sigma_{t^{\prime} t}\right)$. By (FS2), $t^{\prime} \in T_{\alpha_{0}+1}$ for some $\alpha<\kappa$. Let $\pi_{t^{\prime} t}\left(\nu^{\prime}\right)=\nu$ and $s^{\prime}=\left\langle\alpha+1, \nu^{\prime}\right\rangle$. Let $\bar{s}, \bar{t}$ be the direct predecessors of $s^{\prime}$ and $t^{\prime}$ in $\prec$. Set $p^{\prime}=\sigma_{s^{\prime} s}^{-1}(p), q^{\prime}=\sigma_{t^{\prime} t}^{-1}(q)$. Then $p^{\prime}=p^{*}\left(\alpha_{0}+1\right), q^{\prime}=q^{*}\left(\alpha_{0}+1\right)$ by the definition of $p^{*}$. Moreover, $p^{\prime}$ and $q^{\prime}$ are not compatible, because if $r \leq p^{\prime}, q^{\prime}$, then $\sigma_{t^{\prime} t}(r) \leq p, q$ by (FS2). Now, we consider several cases.

Case 1: $\nu^{\prime} \notin r n g\left(\pi_{\bar{t} t^{\prime}}\right)$.

Then $\pi_{\bar{s} s^{\prime}}=i d \uparrow \nu(\bar{s})+1$ and $\sigma_{\bar{s} s^{\prime}}=i d \uparrow \mathbb{P}_{\nu(\bar{s})+1}$ by the minimality of $\alpha_{0}$. Moreover, $\bar{p}:=p^{\prime}$ and $\bar{q}:=e_{\alpha}\left(q^{\prime}\right)$ are not compatible, because if $r \leq p^{\prime}, e_{\alpha}\left(q^{\prime}\right)$, then there is $u \leq r, q^{\prime}, p^{\prime}$ by (FS6)(a). There is no difference between compatibility in $\mathbb{P}_{\theta_{\alpha+1}}$ and in $\mathbb{P}_{\nu\left(t^{\prime}\right)+1}$ by (FS1). Finally, note that $\bar{p}=p^{*}\left(\alpha_{0}\right)$ and $\bar{q}=q^{*}\left(\alpha_{0}\right)$ by the definition of $p^{*}$ and (FS7).

Case 2: $\nu^{\prime} \in \operatorname{rng}\left(\pi_{\bar{t} t^{\prime}}\right)$ and $\pi_{\bar{s} s^{\prime}}=i d \uparrow \nu(\bar{s})+1$. 
Then $\pi_{\bar{t} t^{\prime}} \neq i d \uparrow \nu(\bar{t})+1$ by the minimality of $\alpha_{0}$, and $\bar{p}:=p^{\prime}$ and $\bar{q}:=e_{\alpha}\left(q^{\prime}\right)$ are not compatible (as in case 1). However, $\bar{p}=p^{*}\left(\alpha_{0}\right)$ and $\bar{q}=q^{*}\left(\alpha_{0}\right)$ by the definition of $p^{*}$ and (FS7).

Case 3: $\nu^{\prime} \in \operatorname{rng}\left(\pi_{\overline{t t^{\prime}}}\right), \pi_{\bar{s} s^{\prime}} \neq i d \uparrow \nu(\bar{s})+1$ and $\alpha_{0}+1 \notin \operatorname{supp}(p)$.

Then $\pi_{\bar{t} t^{\prime}} \neq i d \uparrow \nu(\bar{t})+1$ by the minimality of $\alpha_{0}$. Set $\bar{p}:=\sigma_{\bar{s} s^{\prime}}^{-1}\left(p^{\prime}\right)$ and $\bar{q}=e_{\alpha}\left(q^{\prime}\right)$. Then $\bar{p}$ and $\bar{q}$ are not compatible, because if $r \leq \bar{p}, \bar{q}$, then there is $u \leq \sigma_{\alpha}(r), q^{\prime}, p^{\prime}$ by (FS6)(b). However, $\bar{p}=p^{*}\left(\alpha_{0}\right)$ and $\bar{q}=q^{*}\left(\alpha_{0}\right)$ by the definition of $p^{*}$ and (FS7).

Case 4: $\nu^{\prime} \in \operatorname{rng}\left(\pi_{\bar{t} t^{\prime}}\right), \pi_{\bar{s} s^{\prime}} \neq i d\left\lceil\nu(\bar{s})+1\right.$ and $\alpha_{0}+1 \notin \operatorname{supp}(q)$.

Then $\pi_{\bar{t} t^{\prime}} \neq i d\left\lceil\nu(\bar{t})+1\right.$. Set $\bar{q}:=\sigma_{\bar{s} s^{\prime}}^{-1}\left(q^{\prime}\right)$ and $\bar{p}=e_{\alpha}\left(p^{\prime}\right)$. Then $\bar{q}$ and $\bar{p}$ are not compatible, because if $r \leq \bar{p}, \bar{q}$, then there is $u \leq \sigma_{\alpha}(r), p^{\prime}, q^{\prime}$ by (FS6)(b).

Case 5: $\alpha_{0}+1 \in \operatorname{supp}(p) \cap \operatorname{supp}(q)$.

Then $\alpha_{0}+1=\max (\operatorname{supp}(p) \cap \operatorname{supp}(q))$, since $\alpha_{0}+1 \geq \max (\operatorname{supp}(q))$, because by definition $q \in r n g\left(\sigma_{r t}\right)$, where $r \prec t$ and $r \in T_{\max (\operatorname{supp}(q))}$. However, $p^{\prime}=p^{*}\left(\alpha_{0}+1\right)$ and $q^{\prime}=q^{*}\left(\alpha_{0}+1\right)$ are not compatible. Contradiction.

So in case 5 we are finished. If we are in cases 1 - 4, we define recursively $\alpha_{n+1}$ from $p^{*}\left(\alpha_{n}\right)$ and $q^{*}\left(\alpha_{n}\right)$ in the same way as we defined $\alpha_{0}$ from $p$ and $q$. As in the previous proof that $\left\langle\gamma_{n}(p) \mid n \in \omega\right\rangle$ is decreasing, we see that $\left\langle\alpha_{n} \mid n \in \omega\right\rangle$ is decreasing. Hence the recursion breaks off, and we end up in case 5 and get the desired contradiction.

Theorem 4.2. Let $\mu, \kappa>\omega$ be cardinals, $\kappa$ regular. Let $\left\langle\left\langle\mathbb{P}_{\eta} \mid \eta \leq \kappa^{+}\right\rangle,\left\langle\sigma_{s t}\right| s \prec\right.$ $\left.t\rangle,\left\langle e_{\alpha} \mid \alpha<\kappa\right\rangle\right\rangle$ be an FS system along a $(\kappa, 1)$-morass $\mathfrak{M}$. Assume that all $\mathbb{P}_{\eta}$ with $\eta<\kappa$ satisfy the $\mu$-cc. Then $\mathbb{P}_{\kappa^{+}}$does also.

Proof. Let $A \subseteq \mathbb{P}_{\kappa^{+}}$be a set of size $\mu$. Assume by the $\Delta$-system lemma that $\{\operatorname{supp}(p) \mid p \in A\}$ forms a $\Delta$-system with root $\Delta$. Set $\alpha=\max (\Delta)$. Then $\mathbb{P}_{\theta_{\alpha}}$ satisfies the $\mu$-cc by the hypothesis of the lemma. So there are $p \neq q \in A$ such that $p^{*}(\alpha)$ and $q^{*}(\alpha)$ are compatible. Hence $p$ and $q$ are compatible by the previous lemma.

\section{A CCC FORCING that AdDS AN $\omega_{2}$-Suslin treE}

As an application, we construct along an $\left(\omega_{1}, 1\right)$-morass a ccc forcing $\mathbb{P}$ that adds an $\omega_{2}$-Suslin tree.

The natural forcing to do this with finite conditions is Tennenbaum's forcing (see [21): Define $P(\theta)$ as the set of all finite trees $p=\left\langle x_{p},<_{p}\right\rangle, x_{p} \subseteq \theta$, such that $\alpha<\beta$ if $\alpha<_{p} \beta$.

Set $p \leq q$ iff $x_{p} \supset x_{q}$ and $<_{q}=<_{p} \cap x_{q}^{2}$.

For $\theta=\omega_{1}, P(\theta)$ is Tennenbaum's forcing to add an $\omega_{1}$-Suslin tree which satisfies ccc. However, if $\theta>\omega_{1}+1$, then

$$
\begin{aligned}
A=\left\{p \in P(\theta) \mid x_{p}=\right. & \left\{\alpha, \alpha+1, \alpha+2, \omega_{1}, \omega_{1}+1\right\}, \alpha<\omega_{1}, \\
& \left.\alpha<_{p} \alpha+1<_{p} \omega_{1}, \alpha<_{p} \alpha+2<_{p} \omega_{1}+1, \alpha+1 \nless_{p} \alpha+2\right\}
\end{aligned}
$$

is an antichain of size $\omega_{1}$. So $P(\theta)$ does not satisfy the ccc, and in order to thin it out so that it obtains ccc, we have to restrict the possible values of the infima in our trees. 
Let $\pi: \bar{\theta} \rightarrow \theta$ be an order-preserving map. Then $\pi: \bar{\theta} \rightarrow \theta$ induces maps $\pi: \bar{\theta}^{2} \rightarrow \theta^{2}$ and $\pi: P(\bar{\theta}) \rightarrow P(\theta)$ in the obvious way:

$$
\begin{gathered}
\pi: \bar{\theta}^{2} \rightarrow \theta^{2}, \quad\langle\alpha, \beta\rangle \mapsto\langle\pi(\alpha), \pi(\beta)\rangle, \\
\pi: P(\bar{\theta}) \rightarrow P(\theta), \quad\left\langle x_{p},<_{p}\right\rangle \mapsto\left\langle\pi\left[x_{p}\right], \pi\left[<_{p}\right]\right\rangle .
\end{gathered}
$$

If $p \in P(\theta)$, then set

$$
\pi^{-1}[p]:=\left\langle\pi^{-1}\left[x_{p} \cap r n g(\pi)\right], \pi^{-1}\left[<_{p} \cap r n g(\pi)\right]\right\rangle .
$$

Then it is easily seen that $\pi^{-1}[p] \in P(\bar{\theta})$.

Now, let us assume that we restrict the allowed values of the infimum $i_{p}(\alpha, \beta)$ of $\alpha, \beta \in x_{p}$ in the tree $p \in P(\theta)$ to a set $F(\alpha, \beta)$. For $\delta<\theta$, we want to find a reduction of $p \in P(\theta)$ with respect to $i d \uparrow \delta$. Let us look for example at a $p$ with $x_{p}=\{\alpha, \beta\}$ and $<_{p}=\{\langle\alpha, \beta\rangle\}$ such that $\alpha<\delta<\beta$. Then we cannot just take $(i d \uparrow \delta)^{-1}$ as a reduction because we could extend it to a condition $q$ such that $i_{q}(\alpha, \gamma)$ exists for some $\gamma \in \delta$. However, $i_{q}(\alpha, \gamma)$ could have any value in $F(\alpha, \gamma)$, while in a common extension $r$ of $p$ and $q$ we have $i_{r}(\gamma, \beta)=i_{q}(\alpha, \gamma)$ and $i_{r}(\alpha, \beta)$ has to be an element of $F(\alpha, \beta)$. We can solve this problem by taking $s$ with $x_{s}=\left\{\alpha, \beta^{\prime}\right\}$ and $<_{s}=\left\{\left\langle\alpha, \beta^{\prime}\right\rangle\right\}$ as a reduction for some $\beta^{\prime}$ with $F\left(\alpha, \beta^{\prime}\right)=F(\alpha, \beta)$. This leads to the following definition in which the $F(\alpha, \beta)$ are not needed anymore. But they could be introduced as the ranges of the morass maps. A similar problem arises in Baumgartner's and Shelah's forcing to add a thin-very tall superatomic Boolean algebra [1]. They explicitly define a function $F$ as above, which they obtain by historic forcing.

We define our FS system by induction on $\beta \leq \omega_{1}$.

Base Case: $\beta=0$,

Then we need to define only $\mathbb{P}_{1}$. Set $\mathbb{P}_{1}:=P(1)$.

Successor Case: $\beta=\alpha+1$.

We first define $\mathbb{P}_{\theta_{\beta}}$. To do so, let

$$
\begin{gathered}
\mathbb{P}_{\theta_{\beta}}^{\prime}:=\left\{\left\langle x_{p} \cup x_{f_{\alpha}(p)},<_{p} \cup<_{f_{\alpha}(p)}\right\rangle \mid p \in \mathbb{P}_{\theta_{\alpha}}\right\} \\
\cup\left\{\left\langlex_{p} \cup x_{f_{\alpha}(p)}, t c\left(<_{p} \cup<_{f_{\alpha}(p)} \cup\left\{\left\langle\eta, \min \left\{\gamma \in\left[\theta_{\alpha}, \theta_{\alpha+1}\left[\mid \gamma \leq_{f_{\alpha}(p)} f_{\alpha}(\eta)\right\}\right\rangle\right\}\right)\right\rangle\right.\right.\right. \\
\left.\mid p \in \mathbb{P}_{\theta_{\alpha}}, \eta \in x_{p}, \eta<f_{\alpha}(\eta)\right\} .
\end{gathered}
$$

In this definition, $t c(x)$ denotes the transitive closure of the binary relation $x$. As we know from Tennenbaum's original proof, every element of $\mathbb{P}_{\theta_{\beta}}^{\prime}$ is an element of $P\left(\theta_{\beta}\right)$ which extends $p$ and $f_{\alpha}(p)$. This is easily seen.

Now, define

$$
\mathbb{P}_{\theta_{\beta}}:=\left\{p \in P\left(\theta_{\beta}\right) \mid r \leq p \text { for some } r \in \mathbb{P}_{\theta_{\beta}}^{\prime}\right\} .
$$

For $t \in T_{\beta}$ set $\mathbb{P}_{\nu(t)+1}=P(\nu(t)+1) \cap \mathbb{P}_{\theta_{\beta}}$ and $\mathbb{P}_{\lambda}=\bigcup\left\{\mathbb{P}_{\eta} \mid \eta<\lambda\right\}$ for $\lambda \in$ Lim. Let $\sigma_{s t}: \mathbb{P}_{\nu(s)+1} \rightarrow \mathbb{P}_{\nu(t)+1}, p \mapsto \pi_{s t}(p)$.

We still need to define $e_{\alpha}$. If $p \in r n g\left(\sigma_{\alpha}\right)$, then set $e_{\alpha}(p)=\sigma_{\alpha}^{-1}(p)$. If $p \in \mathbb{P}_{\theta_{\alpha}}$, then set $e_{\alpha}(p)=p$. Finally, if $p \notin r n g\left(\sigma_{\alpha}\right) \cup \mathbb{P}_{\theta_{\alpha}}$, then pick an $r \in \mathbb{P}_{\theta_{\beta}}^{\prime}$ such that $r \leq p$ and set $e_{\alpha}(p)=f_{\alpha}^{-1}[r]$.

Limit Case: $\beta \in$ Lim.

Then everything is already uniquely determined by (FS1) and (FS2). That is, for $t \in T_{\beta}$ set $\mathbb{P}_{\nu(t)+1}=\bigcup\left\{\sigma_{s t}\left[\mathbb{P}_{\nu(s)+1}\right] \mid s \prec t\right\}$ and $\mathbb{P}_{\lambda}=\bigcup\left\{\mathbb{P}_{\eta} \mid \eta<\lambda\right\}$ for $\lambda \in \operatorname{Lim}$. Let $\sigma_{s t}: \mathbb{P}_{\nu(s)+1} \rightarrow \mathbb{P}_{\nu(t)+1}, p \mapsto \pi_{s t}(p)$.

Lemma 5.1. $\mathbb{P}$ satisfies the ccc. 
Proof. Since all $P(\theta)$ for $\theta<\omega_{1}$ have size $\leq \omega$, it suffices by Theorem 4.2 to show that $\left\langle\left\langle\mathbb{P}_{\eta} \mid \eta \leq \kappa^{+}\right\rangle,\left\langle\sigma_{s t} \mid s \prec t\right\rangle,\left\langle e_{\alpha} \mid \alpha<\kappa\right\rangle\right\rangle$ is a FS system along the morass.

Most conditions of the definition of an FS system are clear. We only prove (FS6). Let $p \in \mathbb{P}_{\theta_{\beta}}$ and $\beta=\alpha+1$. We may assume that $p \in \mathbb{P}_{\theta_{\beta}}^{\prime}$, because by definition $\mathbb{P}_{\theta_{\beta}}$ is dense in $\mathbb{P}_{\theta_{\beta}}^{\prime}$. We have to prove that $\sigma_{\alpha}^{-1}[p]$ is a reduction of $p$ with respect to $\sigma_{\alpha}$ and $i d \uparrow \mathbb{P}_{\theta_{\alpha}}$. To do so for $\sigma_{\alpha}$, let $q \leq \sigma_{\alpha}^{-1}[p]=: s$. We have to find an $r \leq p, \sigma_{\alpha}(q)$ such that $r \in \mathbb{P}_{\theta_{\beta}}$. We consider two cases. If $p$ is of the form $\left\langle x_{s} \cup x_{f_{\alpha}(s)},<_{s} \cup<_{f_{\alpha}(s)}\right\rangle$, then define $r:=\left\langle x_{q} \cup x_{f_{\alpha}(q)},<_{p} \cup<_{f_{\alpha}(q)}\right\rangle$. It is easily seen that this is an extension of $p$ and $\sigma_{\alpha}(q)$. If $p$ is of the form

$$
\left\langle x_{s} \cup x_{f_{\alpha}(s)}, t c\left(<_{s} \cup<_{f_{\alpha}(s)} \cup\left\{\left\langle\eta, \min \left\{\gamma \in\left[\theta_{\alpha}, \theta_{\alpha+1}\left[\mid \gamma \leq_{f_{\alpha}(s)} f_{\alpha}(\eta)\right\}\right\rangle\right\}\right)\right\rangle\right.\right.
$$

for some $\eta \in x_{s}$, then define $r$ as

$$
\left\langle x_{q} \cup x_{f_{\alpha}(q)}, t c\left(<_{q} \cup<_{f_{\alpha}(q)} \cup\left\{\left\langle\eta, \min \left\{\gamma \in\left[\theta_{\alpha}, \theta_{\alpha+1}\left[\mid \gamma \leq_{f_{\alpha}(q)} f_{\alpha}(\eta)\right\}\right\rangle\right\}\right)\right\rangle .\right.\right.
$$

Again, it is easily seen that this is an extension of $p$ and $\sigma_{\alpha}(q)$. That proves that $\sigma_{\alpha}^{-1}[p]$ is a reduction of $p$ with respect to $\sigma_{\alpha}$. The proof that $\sigma_{\alpha}^{-1}[p]$ is a reduction of $p$ with respect to $i d \uparrow \mathbb{P}_{\theta_{\alpha}}$ is completely analogous.

Lemma 5.2. If $\gamma_{0}(p)=\gamma_{0}(q), p^{*}\left(\gamma_{0}(p)\right)=q^{*}\left(\gamma_{0}(q)\right), \pi: p \cong q$ and $\alpha \leq \pi(\alpha)$, then there exists an $r \leq p, q$ such that $\langle\alpha, \pi(\alpha)\rangle \in \leq_{r}$.

Proof. Let $p$ and $q$ be as in the hypothesis of the lemma. We prove by induction over $\eta \in\left[\gamma_{0}(p), \omega_{1}\right]$ that if $\pi: p^{*}(\eta) \cong q^{*}(\eta)$ (where $p^{*}\left(\omega_{1}\right):=p$ ) and $\alpha \leq \pi(\alpha)$, then there exists an $r \leq p^{*}(\eta), q^{*}(\eta)$ such that $\langle\alpha, \pi(\alpha)\rangle \in \leq_{r}$.

Base Case: $\eta=\gamma_{0}(p)=\gamma_{0}(q)$.

In this case the claim is trivial because $p^{*}(\eta)=q^{*}(\eta)$.

Successor Case: $\eta=\gamma+1$.

Let $\pi: p^{*}(\eta) \cong q^{*}(\eta)$ and $\alpha \leq \pi(\alpha)$. Let $\sigma_{p}: p^{*}(\gamma) \cong p^{*}(\eta), \sigma_{q}: q^{*}(\gamma) \cong q^{*}(\eta)$ and $\sigma_{p}\left(\bar{\alpha}_{p}\right)=\alpha, \sigma_{q}\left(\bar{\alpha}_{q}\right)=\pi(\alpha)$. By the induction hypothesis, there is an $s \leq$ $p^{*}(\gamma), q^{*}(\gamma)$ such that $\left\langle\bar{\alpha}_{q}, \bar{\alpha}_{p}\right\rangle \in \leq_{s}$ or $\left\langle\bar{\alpha}_{p}, \bar{\alpha}_{q}\right\rangle \in \leq_{s}$. Let $\bar{\alpha}:=\max \left\{\bar{\alpha}_{p}, \bar{\alpha}_{q}\right\}$. Now, we consider two cases. If $\bar{\alpha}<f_{\gamma}(\bar{\alpha})$, we define $r$ as

$$
\left\langle x_{s} \cup x_{f_{\alpha}(s)}, t c\left(<_{s} \cup<_{f_{\alpha}(s)} \cup\left\{\left\langle\bar{\alpha}, \min \left\{\beta \in\left[\theta_{\gamma}, \theta_{\gamma+1}\left[\mid \beta \leq_{f_{\alpha}(s)} f_{\gamma}(\bar{\alpha})\right\}\right\rangle\right\}\right)\right\rangle .\right.\right.
$$

If $\bar{\alpha}=f_{\gamma}(\bar{\alpha})$, then we define

$$
r:=\left\langle x_{s} \cup x_{f_{\alpha}(s)},<_{s} \cup<_{f_{\alpha}(s)}\right\rangle .
$$

In both cases, it is easily seen that $r \leq p^{*}(\eta), q^{*}(\eta)$ and $\langle\alpha, \pi(\alpha)\rangle \in \leq_{r}$.

Limit Case: $\eta \in$ Lim.

By (FS1) and (FS2), there are a $t \in T_{\eta}$ and an $s \prec t$ such that $p^{*}(\eta), q^{*}(\eta) \in$ $r n g\left(\sigma_{s t}\right)$. Let $s \in T_{\gamma}, \sigma_{s t}(\bar{\alpha})=\alpha$ and $\sigma_{s t} \circ \bar{\pi}=\pi \circ \sigma_{s t}$. Then $\sigma_{s t}\left(p^{*}(\eta)\right)=p^{*}(\gamma)$ and $\sigma_{s t}\left(q^{*}(\eta)\right)=q^{*}(\gamma)$. Moreover, by the induction hypothesis, there is an $\bar{r} \leq$ $p^{*}(\gamma), q^{*}(\gamma)$ such that $\langle\bar{\alpha}, \bar{\pi}(\bar{\alpha})\rangle \in \leq_{\bar{r}}$. Set $r:=\sigma_{s t}(\bar{r})$. Then $r$ is as desired.

Lemma 5.3. If $\gamma_{0}(p)=\gamma_{0}(q), p^{*}\left(\gamma_{0}(p)\right)=q^{*}\left(\gamma_{0}(q)\right), \pi: p \cong q$ and $\alpha \leq \pi(\alpha)$, then there exists an $r \leq p, q$ such that $\langle\alpha, \pi(\alpha)\rangle \notin<_{r}$.

Proof. Basically the proof proceeds as the proof of Lemma 5.3. However, in the successor case, we always use common extensions of the form $\left\langle x_{p} \cup x_{f_{\gamma}(p)},<_{p} \cup\right.$ $\left.<_{f_{\gamma}(p)}\right\rangle$.

Theorem 5.4. If there is a simplified $\left(\omega_{1}, 1\right)$-morass, then there is a ccc forcing that adds an $\omega_{2}$-Suslin tree. 
Proof. We show that $\mathbb{P}$ forces an $\omega_{2}$-Suslin tree. To do so, we prove that the generic tree has neither an antichain nor a chain of size $\omega_{2}$. First, assume towards a contradiction that there is an antichain of size $\omega_{2}$. Then there is a $p \in \mathbb{P}$ and by ccc of $\mathbb{P}$ a sequence $\left\langle\dot{x}_{i} \mid i \in \omega_{2}\right\rangle$ such that $p \Vdash\left(\left\{\dot{x}_{i} \mid i \in \omega_{2}\right\}\right.$ is an antichain). Let $\left\langle\alpha_{i} \mid i \in \omega_{2}\right\rangle$ and $\left\langle p_{i} \mid i \in \omega_{2}\right\rangle$ be such that $p_{i} \leq p$ for all $i \in \omega_{2}$ and $p_{i} \Vdash\left(\dot{x}_{i}=\check{\alpha}_{i} \wedge \dot{x}_{i} \in \check{x}_{p_{i}}\right)$. Since $\operatorname{card}\left(\mathbb{P}_{\omega_{1}}\right)=\omega_{1}$, there is $q \in \mathbb{P}_{\omega_{1}}, \eta \in \omega_{1}$ and a subset $X \subseteq \omega_{2}$ of size $\omega_{2}$ such that $\gamma_{0}\left(p_{i}\right)=\eta$ and $p_{i}^{*}\left(\gamma_{0}\left(p_{i}\right)\right)=q$ for all $i \in X$. Hence all $p_{i}$ with $i \in X$ are isomorphic. Since $x_{q}$ is finite, there are $i \neq j \in X$ such that $\pi\left(\alpha_{i}\right)=\alpha_{j}$ and $\alpha_{i} \leq \alpha_{j}$, where $\pi: p_{i} \cong p_{j}$. By Lemma 5.2, there exists an $r \leq p_{i}, p_{j}$ such that $\left\langle\alpha_{i}, \alpha_{j}\right\rangle \in \leq_{r}$. Hence $r \Vdash\left(\alpha_{i}\right.$ and $\alpha_{j}$ are comparable $)$. That contradicts the definition of $p$. The proof that there is no chain of size $\omega_{2}$ works the same using Lemma 5.3 instead of Lemma 5.2.

\section{REFERENCES}

1. James E. Baumgartner and Saharon Shelah, Remarks on superatomic Boolean algebras, Ann. Pure Appl. Logic 33 (1987), no. 2, 109-129. MR874021 (88d:03100)

2. Keith J. Devlin, Constructibility, Perspectives in Mathematical Logic, Springer-Verlag, Berlin, 1984. MR 750828 (85k:03001)

3. Hans-Dieter Donder, Another look at gap-1 morasses, Recursion theory (Ithaca, N.Y., 1982), Proc. Sympos. Pure Math., vol. 42, Amer. Math. Soc., Providence, RI, 1985, pp. 223-236. MR791060 (86k:03046)

4. Sy D. Friedman, Fine structure and class forcing, de Gruyter Series in Logic and its Applications, vol. 3, Walter de Gruyter \& Co., Berlin, 2000. MR1780138 (2001g:03001)

5 . Bernhard Irrgang, Constructing $\left(\omega_{1}, \beta\right)$-morasses for $\omega_{1} \leq \beta$, unpublished.

6. _ Proposing $\left(\omega_{1}, \beta\right)$-morasses for $\omega_{1} \leq \beta$, unpublished.

7. K Kondensation und Moraste, dissertation, Universität München, 2002.

8. Ronald B. Jensen, Box implies GKH, hand-written notes.

9.

10. I. Juhász and L. Soukup, How to force a countably tight, initially $\omega_{1}$-compact and noncompact space?, Topology Appl. 69 (1996), no. 3, 227-250. MR.1382294 (97c:54004)

11. Piotr Koszmider, On strong chains of uncountable functions, Israel J. Math. 118 (2000), 289-315. MR1776085 (2001g:03091)

12. Kenneth Kunen, Set theory. An introduction to independence proofs, Studies in Logic and the Foundations of Mathematics, vol. 102, North-Holland Publishing Co., Amsterdam, 1980. MR:597342 (82f:03001)

13. Juan Carlos Martínez, A consistency result on thin-very tall Boolean algebras, Israel J. Math. 123 (2001), 273-284. MR1835300 (2003c:03090)

14. Charles Morgan, Morasses, square and forcing axioms, Ann. Pure Appl. Logic 80 (1996), no. 2, 139-163. MR1402976 (97j:03101)

15. Higher gap morasses. IA. Gap-two morasses and condensation, J. Symbolic Logic 63 (1998), no. 3, 753-787. MR1649060 (2000b:03127)

16. _ Local connectedness and distance functions, Set theory, Trends Math., Birkhäuser, Basel, 2006, pp. 345-400. MR 2267157 (2007h:03104)

17. Mariusz Rabus, An $\omega_{2}$-minimal Boolean algebra, Trans. Amer. Math. Soc. 348 (1996), no. 8, 3235-3244. MR1357881 (96j:03070)

18. R. M. Solovay and S. Tennenbaum, Iterated Cohen extensions and Souslin's problem, Ann. of Math. (2) 94 (1971), 201-245. MR0294139 (45:3212)

19. Lee Stanley, L-like models of set theory: Forcing, combinatorial principles, and morasses, Dissertation, UC Berkeley, 1977.

20. _ A short course on gap-one morasses with a review of the fine structure of $L$, Surveys in set theory, London Math. Soc. Lecture Note Ser., vol. 87, Cambridge Univ. Press, Cambridge, 1983, pp. 197-243. MR823781 (87f:03147)

21. S. Tennenbaum, Souslin's problem, Proc. Nat. Acad. Sci. U.S.A. 59 (1968), 60-63. MR0224456 $(37: 55)$

22. Stevo Todorčević, Coherent sequences, preprint. 
23. Directed sets and cofinal types, Trans. Amer. Math. Soc. 290 (1985), no. 2, 711-723. MR792822 (87a:03084)

24. , Partitioning pairs of countable ordinals, Acta Math. 159 (1987), no. 3-4, 261-294. MR 908147 (88i:04002)

25. - Partition problems in topology, Contemporary Mathematics, vol. 84, American Mathematical Society, Providence, RI, 1989. MR980949 (90d:04001)

26. Boban Veličković, Forcing axioms and stationary sets, Adv. Math. 94 (1992), no. 2, 256-284. MR.1174395 (93k:03045)

27. Dan Velleman, Simplified morasses, J. Symbolic Logic 49 (1984), no. 1, 257-271. MR.736620 (85i:03162)

28. - Souslin trees constructed from morasses, Axiomatic set theory (Boulder, Colo., 1983), Contemp. Math., vol. 31, Amer. Math. Soc., Providence, RI, 1984, pp. 219-241. MR763903 (86b:03066)

29. _ Gap-2 morasses of height $\omega$, J. Symbolic Logic 52 (1987), no. 4, 928-938. MR916398 (88k:03103)

30. , Simplified gap-2 morasses, Ann. Pure Appl. Logic 34 (1987), no. 2, 171-208. MR.890600 (88d:03069)

Mathematisches Institut, Universität Bonn, Beringstrasse 1, 53115 Bonn, Germany 